\title{
Influence of radiation reaction force on ultraintense laser-driven ion acceleration
}

\author{
R. Capdessus* and P. McKenna ${ }^{\dagger}$ \\ Department of Physics SUPA, University of Strathclyde, Glasgow G4 ONG, United Kingdom
}

(Received 3 October 2014; published 19 May 2015)

\begin{abstract}
The role of the radiation reaction force in ultraintense laser-driven ion acceleration is investigated. For laser intensities $\sim 10^{23} \mathrm{~W} / \mathrm{cm}^{2}$, the action of this force on electrons is demonstrated in relativistic particle-in-cell simulations to significantly enhance the energy transfer to ions in relativistically transparent targets, but strongly reduce the ion energy in dense plasma targets. An expression is derived for the revised piston velocity, and hence ion energy, taking account of energy loses to synchrotron radiation generated by electrons accelerated in the laser field. Ion mass is demonstrated to be important by comparing results obtained with proton and deuteron plasma. The results can be verified in experiments with cryogenic hydrogen and deuterium targets.
\end{abstract}

DOI: 10.1103/PhysRevE.91.053105

\section{INTRODUCTION}

Peak laser pulse intensities of $\sim 10^{21} \mathrm{~W} / \mathrm{cm}^{2}$ are presently achievable at petawatt-scale laser facilities and the next generation of multipetawatt lasers under construction aims to push the intensity frontier beyond $10^{23} \mathrm{~W} / \mathrm{cm}^{2}$. Among many research topics to be explored using these sources, the acceleration of ions to high energies suitable for applications in cancer therapy, radiography, and fast ignition [1] is of high priority. At these ultrahigh intensities the radiation pressure acceleration (RPA) mechanism should enable efficient acceleration of ions to hundreds-of-MeV energies, in a peaked-energy spectrum and a narrow divergence beam [2-5]. However, recent theoretical and numerical studies also show that the radiation reaction (RR) force acting on energetic electrons in the high laser fields produced at these intensities will strongly impact on the electron dynamics [6-10] and thus ion acceleration due to modification of the induced charge-separation fields.

The effects of $\mathrm{RR}$ on ion acceleration are explored in several numerical studies. Chen et al. [9] predicts an ion energy enhancement with thin targets due to modification of the electron phase space, by modeling the interaction of a trapezoidal-temporal-profile laser pulse. This effect is expected to be less important in the case of a Gaussian pulse, which is typically achieved experimentally. Naumova et al. [11] show that radiation losses curb the electron backward motion, which reinforces the electron bunch structuring at the front of the laser field, thus affecting the hole-boring mode [4] of RPA. The use of linearly polarized laser light is also reported to enhance the RR energy losses compared to circular polarization in the case of a thin, dense foil, by Tamburini et al. [8]. The modifications to ion acceleration suggested by these first studies highlights the need for a detailed investigation into the role of RR effects on the physics of ion acceleration. To date, the significant percentage of the laser energy converted into high energy synchrotron radiation at laser intensities $\sim 10^{23} \mathrm{~W} / \mathrm{cm}^{2}$ is not accounted for in calculations of the piston velocity used to predict RPA-ion energies.

In this article, we report on the role of $R R$ in ion acceleration in the relativistic self-induced transparency regime and in the transition to the hole-boring RPA regime. Using particle

\footnotetext{
*remi.capdessus@strath.ac.uk

†paul.mckenna@strath.ac.uk
}

PACS number(s): 52.38.Ph, 41.60.-m, 52.65.Rr

in-cell (PIC) simulations, we demonstrate that the ion energy is enhanced in the case of relativistically transparent targets, but that the piston velocity and subsequently ion energies are reduced for thicker, opaque targets. We also derive a first (to our knowledge) analytical model to account for the effect of the energy radiated on the piston velocity and this is shown to be in good agreement with the simulation results. The importance of collective effects on RR and ion acceleration is also demonstrated, by comparing results for deuteron and proton plasma.

\section{UNDERPINNING THEORY}

\section{A. Electron motion equations}

The RR force is described using the model developed by Sokolov, where the RR force has been derived from QED principles and can be extended to QED regimes [12-14], contrary to the Landau-Lifshitz (LL) equation [15]. It is close to the LL equation for classical regimes, i.e., $\chi_{e} \leqslant 1$, where the parameter,

$$
\chi_{e}=\frac{\gamma_{e} \sqrt{\mathbf{F}_{\mathrm{Le}}^{2}-\left(\mathbf{F}_{\mathrm{Le}} \cdot \boldsymbol{\beta}_{e}\right)^{2}}}{\left(e E_{\mathrm{sh}}\right)},
$$

defines the ratio between the laser electric field and the Schwinger field, $E_{\mathrm{sh}}=m_{e} c^{2} / e \lambda_{c}$ (where $\lambda_{c}=h / m_{e} c$ is the Compton wavelength), in the electron frame of reference. Here, $\mathbf{F}_{\mathrm{Le}}=-e\left(\mathbf{E}+\mathbf{v}_{e} \times \mathbf{B}\right)$ is the Lorentz force and $\tau_{r}=$ $e^{2} / 6 \pi \epsilon_{0} m_{e} c^{3} \simeq 6.2 \times 10^{-24} \mathrm{~s}$ is the characteristic radiation time, where $e$ and $m_{e}$ are the electron charge and mass, respectively, $c$ is the velocity of light, $\omega_{L}$ is the laser frequency, and $\gamma_{e}$ is the electron Lorentz factor. The Sokolov equations are easier to implement in a PIC code than the LL equation and conserve the four-momentum. The electron motion equations are

$$
\begin{gathered}
\frac{d \mathbf{p}_{e}}{d t}=\mathbf{F}_{\mathrm{Le}}+c \delta \boldsymbol{\beta}_{e} \times \mathbf{B}-\gamma_{e}^{2}\left(\mathbf{F}_{\mathrm{Le}} . \delta \boldsymbol{\beta}_{e}\right) \boldsymbol{\beta}_{e}, \\
\frac{d \mathbf{x}_{e}}{d t}=c \boldsymbol{\beta}_{e}+c \delta \boldsymbol{\beta}_{e},
\end{gathered}
$$

where

$$
\delta \boldsymbol{\beta}_{e}=\frac{\tau_{r}}{m_{e} c} \frac{\mathbf{F}_{\mathrm{Le}}-\left(\mathbf{F}_{\mathrm{Le}} \cdot \boldsymbol{\beta}_{e}\right) \boldsymbol{\beta}_{e}}{1+\frac{\tau_{r}}{m_{e} c}\left(\mathbf{F}_{\mathrm{Le}} \cdot \boldsymbol{\beta}_{e}\right)}
$$


is the radiation correction to the electron velocity typical of the Sokolov model. The influence of this perturbative velocity is negligible on ion acceleration for $\chi_{e} \leqslant 1[10]$.

\section{B. The synchrotron radiation}

The energetic synchrotron radiation produced in the interaction of an ultraintense laser pulse with a plasma is directly related to the RR force as follows:

$$
\begin{aligned}
\frac{d^{2} P_{r}}{d \omega d \boldsymbol{\Omega}}= & \gamma_{e}^{2} \frac{\tau_{r}}{m_{e}} \mathbf{F}_{\mathrm{Le}}^{2}\left(1-\cos ^{2} \psi \beta_{e}^{2}\right) \boldsymbol{\delta}\left(\boldsymbol{\Omega}-\frac{\mathbf{p}_{e}}{\left\|\mathbf{p}_{\mathbf{e}}\right\|}\right) \\
& \times S\left(\frac{\omega}{\omega_{\mathrm{cr}}}\right),
\end{aligned}
$$

where $S(r)$ describes the normalized spectral shape, and $\omega_{\mathrm{cr}}=$ $3 / 2 \gamma_{e}^{3}\left\|\mathbf{p}_{e} \times \mathbf{F}_{\mathrm{Le}}\right\| / p_{e}^{2}$ is the critical frequency [15]. Here, $\psi$ is the angle between the laser electric field and the electron velocity. Originally the synchrotron radiation corresponds to the emission generated by an electron in a homogeneous magnetic field. Here, this notion is applied in a more general sense to the emission generated by a relativistic electron in the combined laser and self-consistent plasma electromagnetic fields. The total power radiated by the electrons from a unit volume of plasma can be written as

$$
\mathcal{W}_{\gamma}=\iint_{0}^{4 \pi} \int f_{e} \frac{d^{2} P_{r}}{d \omega d \boldsymbol{\Omega}} d \omega d \boldsymbol{\Omega} d \mathbf{p}_{e},
$$

where $f_{e}$ is the electron momentum distribution with $\int f_{e} \mathbf{d p}_{e}=n_{e}$. In the case of a Maxwellian-Juttner distribution with $a_{L} \gg 1$ and assuming that $T_{e} \sim a_{L} m_{e} c^{2}$ [16], Eq. (6) reduces to $[17,18]$

$$
\mathcal{W}_{\gamma} \simeq 6 n_{e}\left(g+\alpha^{2}\right) a_{L}^{4} \tau_{r} \omega_{L} m_{e} c^{2} \omega_{L},
$$

where $\int_{0}^{\infty} g(t) d t \equiv \tau_{L}$ is the laser-pulse duration and $\alpha \equiv$ $E_{S} / E_{L}$ is the ratio of the electrostatic field to the laser field.

\section{NUMERICAL APPROACH}

\section{A. Accounting for the radiation}

The PICLS 1D PIC code [19], which has recently been upgraded to include the RR force and the synchrotron radiation $[10,20]$, is used. Although laser energy coupling to ions is typically overestimated in 1D PIC simulations (due to the fact that transverse effects are not accounted for), this is not an issue in determining whether the RR force increases or decreases the ion energy. The fundamental RR physics is independent of the dimension of the simulations, although the overall magnitude of the effects of the RR force on ion acceleration may be reduced in simulations at higher dimensions. Importantly, the use of 1D simulations enables the fundamental physics of RR to be explored in plasma free from transverse effects such as instabilities, thereby decoupling the RR physics from such effects.

We use a fourth-order interpolation for the numerical solver presented in Ref. [19], to apply fields and to deposit currents. The time step $\Delta t$ is linked to the mesh size $\Delta x$ by a simple relation: $\Delta x=c \Delta t$, where $\mathrm{c}$ is the light velocity. The numerical implementation of radiation losses has been discussed in Refs. [8] and [10]. The radiation is computed from the macroparticle trajectories assuming the emission to be incoherent due to the fact the average wavelength $\lambda_{\gamma}$ of the intense radiation is much smaller than the characteristic distance between electrons, $d$ :

$$
\lambda_{\gamma} \sim \frac{2 \pi c}{\omega_{\mathrm{cr}}} \ll d \sim n_{e}^{-1 / 3}
$$

In the frame of the synchrotron radiation, the electron trajectory at each time step can be approximated by an arc [15], ensuring that the angular variation of the electron momentum is less than $1 / \gamma_{e}$ at the computational time step $\Delta t$. Considering the instantaneous electron rotation frequency [15], $\omega_{\text {re }}=$ $\left\|\mathbf{p}_{e} \times \mathbf{F}_{\mathrm{Le}}\right\| / p_{e}^{2} \sim \frac{a_{L}}{\gamma_{e}} \omega_{L}$, this imposes the following condition [17]:

$$
\Delta t \leqslant \frac{T_{L}}{a_{L}} .
$$

We consider a grid of 1000 cells in the photon energy over the range $10^{-3} \mathrm{MeV} \leqslant \hbar \omega \leqslant 10^{3} \mathrm{MeV}$ in agreement with the incoherence condition of the radiation [Eq. (8)], 90 cells in the polar angle $\theta$ over the range $0^{\circ} \leqslant \theta \leqslant 360^{\circ}$. The polar axis is defined along the laser propagation direction. These numerical parameters enable a compromise between good precision, minimum noise, and reasonable calculation time.

The validity of our classical approach has been confirmed in Ref. [17], where it is shown that the number of quantum electrons $\left(\chi_{e} \geqslant 0.2\right)$ represents a small part (few percent) of the electron population and do not significantly affect the electron dynamics and the synchrotron radiation emission. This is in agreement with the discussion of this issue in Refs. [21,22]. This implies that we can reasonably assume that the plasma is transparent to the intense synchrotron radiation generated by the ultrarelativistic electrons, due to the fact the absorption rate of such radiation depends on the parameter $\chi_{e}$. The absorption rate of this intense radiation becomes nonnegligible for electrons with $\chi_{e} \gtrsim 1$ [23-25] (which is not the case in the present study).

\section{B. Simulation parameters}

To investigate the role of $\mathrm{RR}$ in ion acceleration, we consider a circularly polarized laser pulse with the dimensionless amplitude vector potential, $a_{L}(t, x)=$ $g(t) \operatorname{Re}(\mathbf{y}-i \mathbf{z}) \exp \left[-i \omega_{L}(t-x / c)\right]$, normally incident on a plasma layer, the thickness, $l$, of which is varied in the range $0.1 \lambda_{L}$ to $100 \lambda_{L}$. Time is considered in units of the laser period $T_{L}=2 \pi / \omega_{L}$ and length in units of laser wavelength $\lambda_{L}=c T_{L}$. As the laser pulse profile is important to the evolving plasma dynamics, a realistic Gaussian laser pulse is considered, with a full width at half maximum (FWHM) duration equal to $13 T_{L}$ (full width $\sim 30 T_{L}=2 T_{\text {rise }}$ ). The target is a deuteron plasma with initial density equal to $10 n_{c}$, where $n_{c}=m_{e} \epsilon_{0} \omega_{L}^{2} / e^{2}$ is the critical density. In this density regime there is strong conversion of the laser energy into intense radiation, as shown in Ref. [20], which means that the RR force strongly effects the plasma dynamics. In accordance with condition Eq. (9), the cell size is $\lambda_{L} / 200$ and each cell contains 100 macroparticles (electrons and ions). The laser pulse starts interacting with the target at $t=0$. The laser amplitude is $a_{L} \equiv \frac{e E_{L}}{m_{e} c \omega_{L}}=$ $a_{x}=a_{y}=200$, which corresponds to an energy fluence of 

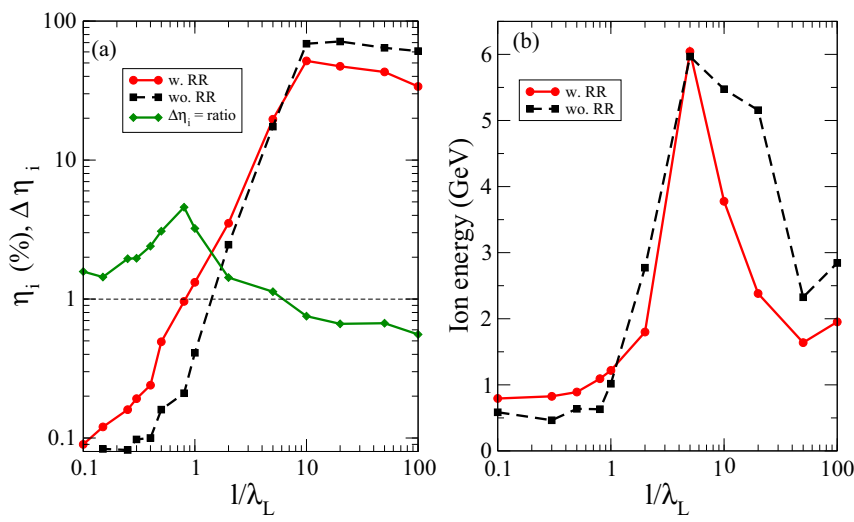

FIG. 1. (Color online) (a) Energy transfer to ions (total ion energy normalised to laser energy) as a function of target thickness: red (circles), with RR; black (squares), without RR; green (diamonds), ratio of the two. (b) Maximum ion energy as a function of target thickness.

$5 \times 10^{9} \mathrm{~J} / \mathrm{cm}^{2}$ and peak intensity equal to $1.1 \times 10^{23} \mathrm{~W} / \mathrm{cm}^{2}$ (which avoids significant QED effects [21,22]). The energetics of the laser-plasma interaction are defined by the parameters $\eta_{k}=\mathcal{E}_{k} / \mathcal{E}_{L}$, where $\mathcal{E}_{L}$ is the laser pulse energy and the subscript $k$ denotes photons $(\gamma)$, electrons $(e)$, or ions $(i)$, i.e., $\mathcal{E}_{\gamma}$ is the energy fluence of the photons radiated, and $\mathcal{E}_{e}$ and $\mathcal{E}_{i}$ are the electron and ion areal energies, respectively, all at time $t$.

\section{ION ACCELERATION}

Figure 1(a) shows simulation results for the laser energy transfer to ions as a function of target thickness. Inclusion of the RR force is found to enhance the ion acceleration for target thickness $l$ up to $5 \lambda_{L}$, with the largest enhancement at $l=0.8 \lambda_{L}$, for which the energy transfer to ions is almost four times higher than in the corresponding case without RR.
Figure 1(b) shows the maximum ion energy as a function of $l$, and the largest enhancement is again obtained for $l=0.8 \lambda_{L}$.

To explore the underlying physics we consider three target thickness cases for which the ratio of the electrostatic pressure, $P_{s}=\frac{1}{2 \epsilon_{0}}\left(e n_{e} l\right)^{2}$, to the laser radiation pressure, $P_{r}=$ $2 \mathcal{R} I_{L} / c=2 \mathcal{R} n_{c} m_{e} c^{2} a_{L}^{2}$ ( $\mathcal{R}$ being the reflection coefficient of the laser pulse), are significantly different, and for which the effects of RR on ion acceleration are different. These are: (1) $l=0.8 \lambda_{L}$, for which $P_{s}<P_{r}$; (2) $l=100 \lambda_{L}$, for which $P_{s}>P_{r}$; and (3), an intermediate case, $l=5 \lambda_{L}$, for which $P_{s} \sim P_{r}$. These cases correspond to three different regimes of ion acceleration, as discussed below. For each case, the efficiency of laser energy transfer to electrons and synchrotron radiation as a function of time are presented in Fig. 2, and the electron and ion phase space and spectra (in both the forward and backward directions) at several moments in time are presented in Figs. 3 and 4, respectively. Note that for the case of the thick target when the RR force is not included [black dashed line in Fig. 2(f)] $\eta_{\gamma}>100 \%$; i.e., energy is not conserved. This emphasizes the need to account for the RR force in the electron equations of motion.

\section{A. The relativistic self-induced transparency regime}

The $l=0.8 \lambda_{L}$ target is relativistically transparent to the laser pulse due to the low electrostatic pressure. For $0.8 \leqslant$ $t / T_{L} \leqslant 30$ the whole target thickness is included within the laser pulse "length," and hence laser energy is efficiently coupled to electrons, as shown in Fig. 2(a). The laser ponderomotively drives electrons forward with longitudinal momentum $p_{\|} \sim a_{L}$, as shown in Fig. 3(a), and these electrons are weakly affected by the RR because they propagate in the same direction as the laser. By contrast, electrons expanding in the backward direction (i.e., counterpropagating with the laser pulse) experience a laser field of amplitude $\simeq 2 \gamma_{e} E_{L}$ in their reference frame, due to the relativistic Doppler effect. These electrons lose a significant portion of their energy to synchrotron radiation and as a result are efficiently reflected

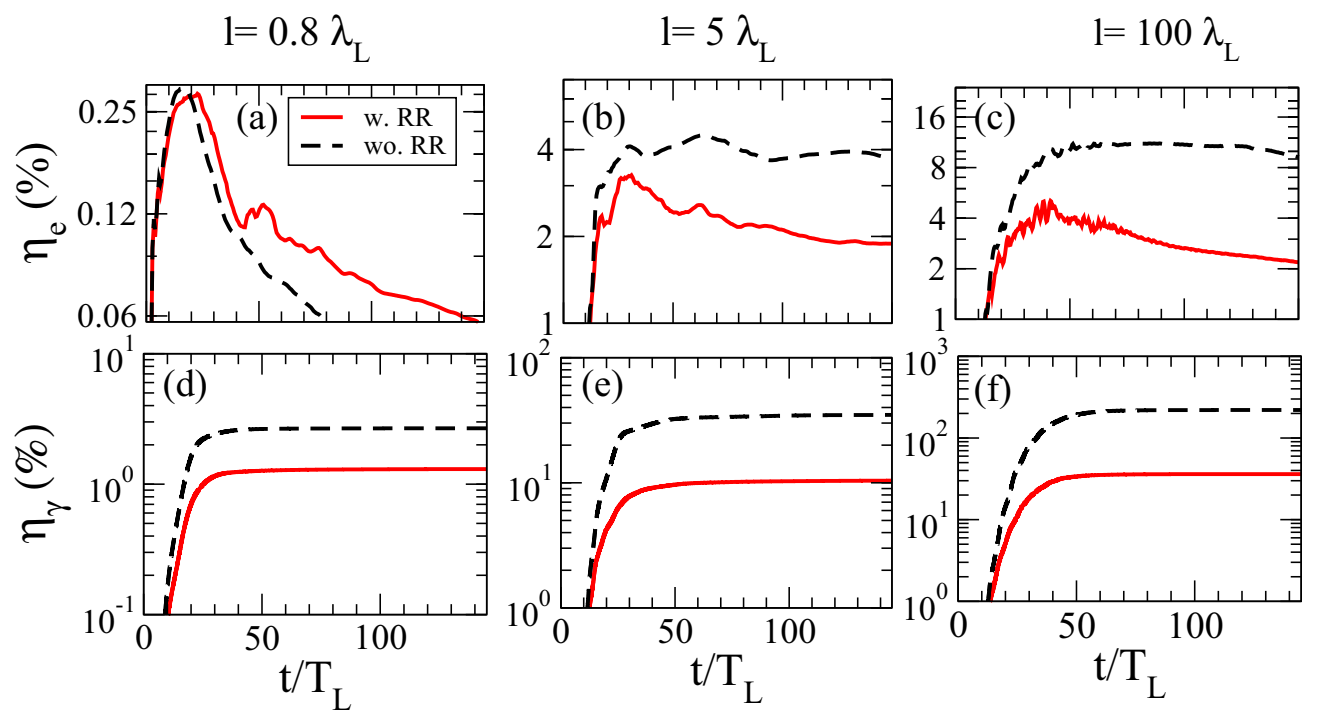

FIG. 2. (Color online) Total electron energy normalized to laser energy, $\eta_{e}$, as a function of time, for (a) $l=0.8 \lambda_{L}$; (b) $l=5 \lambda_{L}$; (c) $l=100 \lambda_{L}$. (d)-(f) Corresponding plots for radiated energy normalized to laser energy, $\eta_{\gamma}$. 


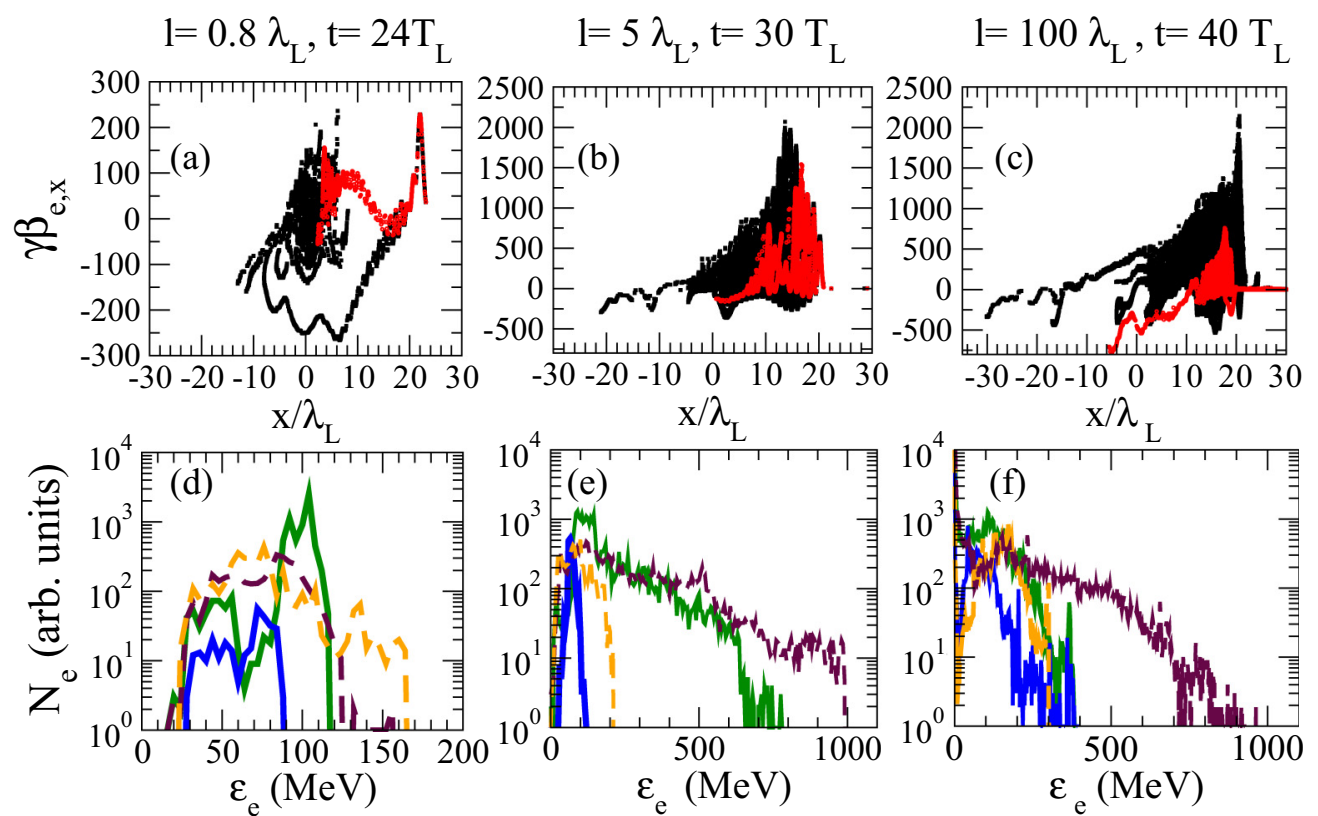

FIG. 3. (Color online) Longitudinal electron phase space for (a) $l=0.8 \lambda_{L}$, (b) $l=5 \lambda_{L}$, and (c) $l=100 \lambda_{L}$ at $t$ such that $\partial \eta_{e} / \partial t \approx 0$. Red, with RR; black, without RR. (d)-(f) Corresponding electron energy spectra: green (thin solid) line, forward electrons with RR; brown (thick dashed) line, forward electrons without RR; blue (thick solid) line, backward electrons with RR; yellow (thin dashed), backward electrons without RR.
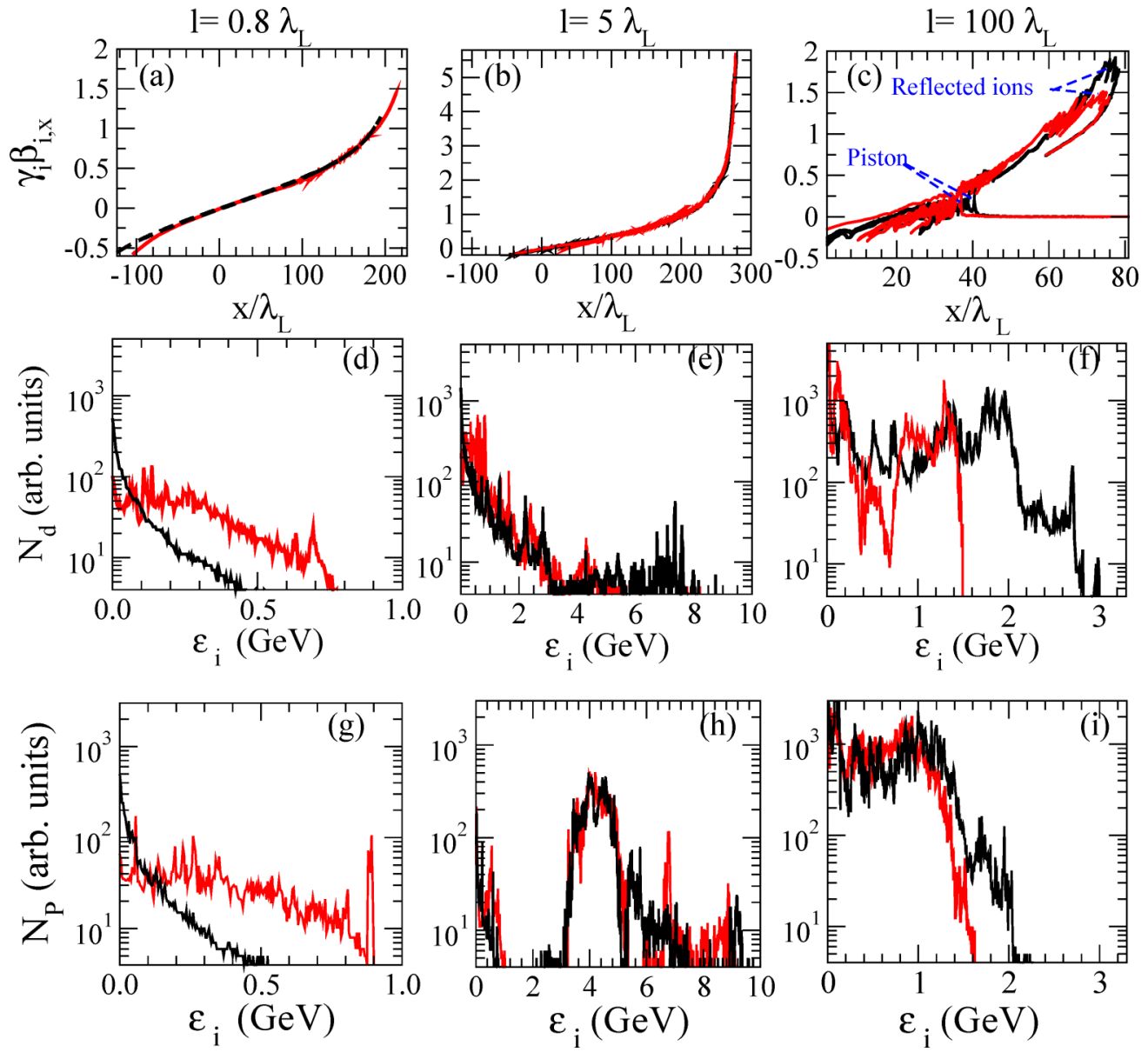

FIG. 4. (Color online) Longitudinal ion phase space for (a) $l=0.8 \lambda_{L}$, (b) $l=5 \lambda_{L}$, and (c) $l=100 \lambda_{L}$ at $t$ such that $\partial \eta_{i} / \partial t \approx 0$ : red, with RR; black, without RR. (d)-(f) Corresponding forward-directed deuteron energy spectra. (g)-(i) Corresponding forward-directed proton energy spectra. Color code: red, with RR; black, without RR. 

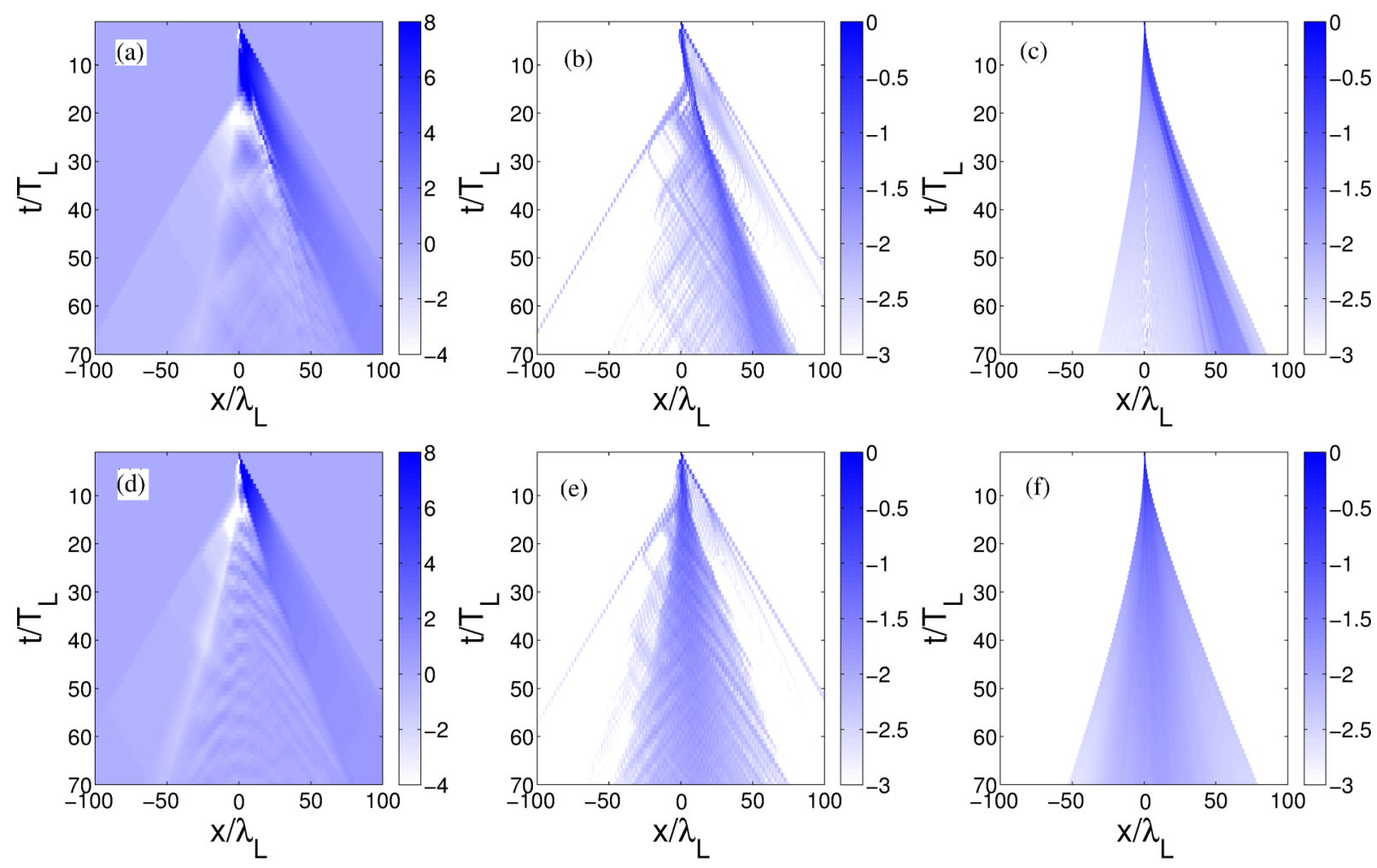

FIG. 5. (Color online) Space-time plots of (a) $\frac{e E_{x}}{m_{e} c \omega_{L}}$ (linear scale), (b) electron, and (c) ion densities [in logarithmic scale: $\left.\ln \left(\frac{n}{n_{0}}\right)\right]$ for $l=0.8 \lambda_{L}$, with RR included. (d)-(f) The corresponding plots without RR inclusion.

forward by the laser field [7] and trapped by the electrostatic sheath field at the boundary of the expanding target, as shown in Fig. 5(b). A compression of the electron phase-space results [26], leading to a reduced electron temperature $T_{e}$ and electron energy spread, as shown in Fig. 3(d). As Fig. 5(a) illustrates, this cooling down tends to increase the electron density at the rear of the target, enhancing the gradient of the electrostatic field. The maximum ion density is increased by $50 \%$ due to the RR. Moreover, during this stage the ions at the target front surface $(x<0)$ are accelerated by a positive electrostatic field, forward with respect to the laser field direction, as shown in Fig. 5(a). This is due entirely to the RR effect. This also maintains the ion density at a value higher than $n_{c}$ for longer, as seen by comparing Figs. 5(c) and 5(f). In other words, the dynamics of the front surface target is no longer governed by the ion velocity $c_{s}=\sqrt{\frac{Z k_{B} T_{e}}{m_{i}}}$. All of these aspects contribute to enhancing the ion acceleration in the forward direction.

After this initial stage, when the laser pulse has passed (i.e., at $t>30 T_{L}$ ), the expansion velocity of the target front surface increases because electrons escaping the target (with $p_{\|} \leqslant 0$ ) are no longer interacting with the laser field and thus losing energy to synchrotron radiation. This behavior is observed when comparing the electron density evolution with [Fig. 5(b)] and without [Fig. 5(e)] RR inclusion.

As the target expansion depends on the ion sound velocity $c_{s}$ and thus the electron temperature $T_{e}$, the target expansion remains slower even after that laser pulse has passed, compared to the case without RR, as observed in Fig. 5. The total radiated energy fluence is close to $1 \%$ of the laser fluence, as shown in Fig. 2(d). The maximum ion energy and flux at high energies are significantly enhanced by the RR force acting on the electrons, and a spectral peak begins to emerge at the maximum energy, as shown in Fig. 4(d).

\section{B. The hole-boring regime}

For a much thicker $l=100 \lambda_{L}$ target $\left(P_{s}>P_{r}\right)$, the electrostatic field produced as electrons are driven forward by the ponderomotive force (during hole-boring) can attain the same magnitude as the laser field [20], giving rise to electron backward motion, and thus to intense synchrotron radiation [27]. Electron cooling due to RR thus plays an important role in defining the electron dynamics, as shown in Fig. 3(c). The electrostatic field propagates with piston velocity $c \boldsymbol{\beta}_{p}$, leading to the forward acceleration of ions, as observed in Fig. 4(c)-this corresponds to ion acceleration in the "piston" or "hole-boring-RPA" regime [4,28]. As little as $4 \%$ of the laser energy is converted into the final electron kinetic energy [see Fig. 2(c)]. To first order, the electron energy has no effect on the piston velocity and can be neglected. However, more than $30 \%$ of the laser energy is converted into high energy synchrotron radiation [see Fig. 2(f)], which has a significant effect on the piston velocity. Below, we derive an analytical expression to quantify this. By following the same procedure as that in references $[4,28,29]$ the energy flux conservation is written as

$$
(1-\mathcal{R})\left(1-\beta_{p}\right) I_{L}=\mathcal{W}_{\gamma}+\left(\gamma_{i}-1\right) M n_{i} \beta_{p} c^{3},
$$

where $M=Z m_{e}+m_{i}$ and $\gamma_{i}=1 /\left(1-\beta_{i}^{2}\right)^{1 / 2}=\left(1+\beta_{p}^{2}\right) /$ $\left(1-\beta_{p}^{2}\right)$ is the relativistic factor of the ions reflected by the piston. The first term on the right-hand side of Eq. (10) is the 
radiated power [Eq. (7)]. The second term on the right-hand side of Eq. (10) expresses the bulk electrons (included in factor $M$ ) and ions reflected from the piston. In a similar way, the flux conservation can be expressed as

$$
(1+\mathcal{R})\left(1-\beta_{p}\right) \frac{I_{L}}{c}=\mathcal{P}_{\gamma}+M c^{2} \gamma_{i} \beta_{p} \beta_{i},
$$

where $\mathcal{P}_{\gamma}=\mathcal{E}_{\gamma} \cos \langle\theta\rangle$ is the pressure of the synchrotron radiation.

By expressing laser intensity as $I_{L}=n_{c} m_{e} c^{3} a_{L}^{2}$ and introducing the dimensionless shock velocity scale [29],

$$
B=\left(\frac{n_{c}}{n_{i}} \frac{m_{e}}{Z m_{e}+m_{i}}\right)^{1 / 2} a_{L},
$$

Eqs. (10) and (11) reduce to

$$
\begin{aligned}
& (1-\mathcal{R})\left(1-\beta_{p}\right)=\left\langle\mathcal{E}_{\gamma}\right\rangle+2 \frac{\beta_{p}^{3}}{B^{2}} \gamma_{p}^{2}, \\
& (1+\mathcal{R})\left(1-\beta_{p}\right)=\left\langle\mathcal{P}_{\gamma}\right\rangle+2 \frac{\beta_{p}^{2}}{B^{2}} \gamma_{p}^{2},
\end{aligned}
$$

where

$$
\begin{aligned}
\left\langle\mathcal{E}_{\gamma}\right\rangle \equiv & \frac{\int_{0}^{\infty} \mathcal{W}_{\gamma} d t}{\mathcal{E}_{L}} \lambda_{L} \simeq 12 \pi a_{L}^{2}\left(\omega_{L} \tau_{r}\right) \frac{n_{e}}{n_{c}} \\
& \times\left\{\frac{1}{\sqrt{2}}+\operatorname{Max}[\alpha]^{2}\left[\frac{T_{\text {rise }}}{\sqrt{\tau_{L}^{2}+T_{\text {rise }}^{2}}}+\frac{T_{\text {rise }}}{\tau_{L} \sqrt{2}}\right]\right\}
\end{aligned}
$$

is the fraction of laser energy converted into synchrotron radiation. Equation (15) is derived from Eq. (7) in Ref. [17]. We put

$$
\left\langle\mathcal{P}_{\gamma}\right\rangle=\left\langle\mathcal{E}_{\gamma}\right\rangle \cos \langle\theta\rangle .
$$

The average angle of emission of the synchrotron radiation, $\langle\theta\rangle$, can be defined as

$$
\cos \langle\theta\rangle \equiv \cos (\langle\boldsymbol{\Omega}\rangle, \mathbf{k})=\frac{\int_{\mathbb{R}^{3}} f_{e} p_{e, x} \mathbf{d} \mathbf{p}_{e}}{\int_{\mathbb{R}^{3}} f_{e}\left\|\mathbf{p}_{e}\right\| \mathbf{d} \mathbf{p}_{e}} .
$$

In order to take into account energy conservation, and thus the saturation of the radiated energy above $I_{L} \simeq 5 \times 10^{23} \mathrm{~W} / \mathrm{cm}^{2}$ observed in simulations (see Ref. [20]), we add to $\left\langle\mathcal{E}_{\gamma}\right\rangle$ a saturation coefficient evolving as

$$
\left\langle\mathcal{E}_{\gamma}\right\rangle \rightarrow\left[1-\exp \left(-A^{2} / a_{L}^{2}\right)\right]\left\langle\mathcal{E}_{\gamma}\right\rangle .
$$

The value of parameter $A$ in Eq. (18) is determined from a fit to the simulation data $(A \simeq 300)$ and not from an analytical model. An investigation of the phenomenon of saturation of the synchrotron radiation is beyond the scope of this article and will be the subject of a separate study.

By subtracting Eq. (14) from Eq. (13), an expression for the reflection coefficient $\mathcal{R}$ is obtained:

$$
\mathcal{R}=\frac{1-\beta_{p}}{1+\beta_{p}}-\frac{(1-\cos \langle\theta\rangle)\left\langle\mathcal{E}_{\gamma}\right\rangle}{2\left(1-\beta_{p}\right)} .
$$

It is difficult to formally evaluate the average angle $\langle\theta\rangle$ due to the complexity of the radiation distribution which strongly depends on the electron dynamics and on the charge separation field, which is a function of ion mass [20]. However, the average angle $\langle\theta\rangle$ can be estimated from the angular

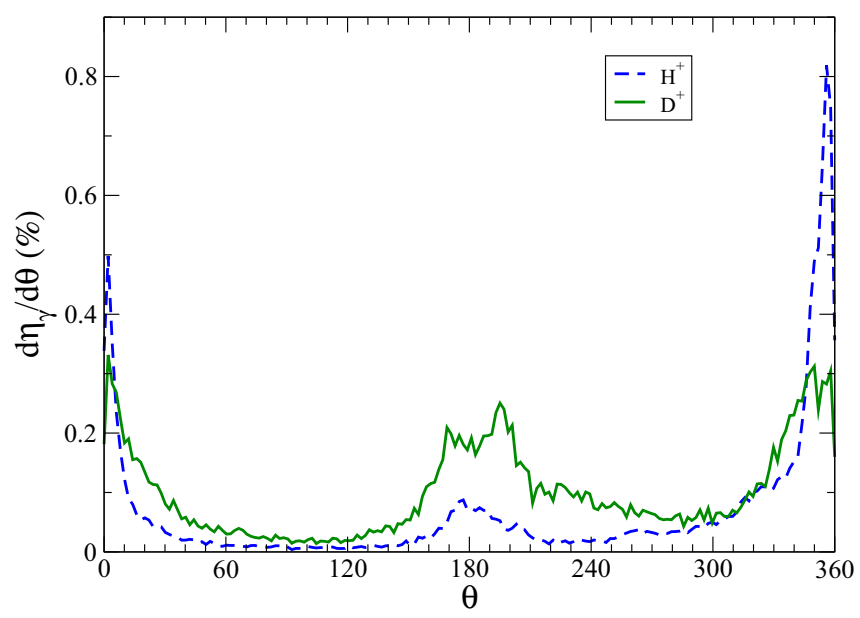

FIG. 6. (Color online) Angular distribution of the synchrotron radiation as a function of the polar angle $\theta$ at $t$ such that $\partial \eta_{\gamma} / \partial t \approx 0$. Green, deuteron plasma; dashed blue, proton plasma.

distribution of the synchrotron radiation resulting from the numerical simulations. In the case of a deuteron plasma, the emitted radiation is mainly distributed between the backward $\left(\theta \simeq 180^{\circ}\right)$ and forward $\left(\theta \simeq\left\{0^{\circ}, 360^{\circ}\right\}\right)$ directions, as shown in Fig. 6. Therefore, the average angle $\langle\theta\rangle$ is close to $90^{\circ}$, which implies that $\cos \langle\theta\rangle \ll 1$. Thus, from Eq. (16), the synchrotron radiation pressure does not have a strong impact on the reflection coefficient and can be neglected to first approximation. By contrast, in the case of a proton plasma the radiation is mainly produced by electrons that propagate forward and radiate in the laser pulse leading to $\cos \langle\theta\rangle \sim 1$. As a consequence, the RR force has a very small effect on the reflection coefficient $\mathcal{R}$ and thus on the piston velocity. Compared to deuterons, the RR force therefore has less effect on the proton energy spectra, as shown in Fig. 4(f).

For simplicity we shall consider the case $\cos \langle\theta\rangle=0$ and use the expression of the reflection coefficient $\mathcal{R}$ in order to deduce an expression for the RR effect on the piston velocity. We note that $\mathcal{R}$ depends on the emitted radiation energy, meaning that the laser energy contributing to the piston drive is reduced. By substituting Eq. (19) into Eq. (14), it can be shown that the expression for the piston velocity $\beta_{p}$ for $\langle\theta\rangle \simeq 90^{\circ}$ (deuteron plasma):

$$
\beta_{p,\langle\theta\rangle \simeq 90^{\circ}}=\frac{B}{B+1} \mathcal{F}\left(\left\langle\mathcal{E}_{\gamma}\right\rangle, B\right)_{\langle\theta\rangle \simeq 90^{\circ}},
$$

where

$$
\begin{aligned}
\mathcal{F}\left(\left\langle\mathcal{E}_{\gamma}\right\rangle, B\right)_{\langle\theta\rangle \simeq 90^{\circ}} \\
\equiv \frac{B-\sqrt{B^{2}-\left[B^{2}-1\right]\left[1+\frac{B^{2}}{4\left(B^{2}-1\right)}\left\langle\mathcal{E}_{\gamma}\right\rangle\right]\left[1-\frac{\left\langle\mathcal{E}_{\gamma}\right\rangle}{4}\right]}}{\left[1+\frac{B^{2}}{4\left[B^{2}-1\right]}\left\langle\mathcal{E}_{\gamma}\right\rangle\right][B-1]} .
\end{aligned}
$$

$\mathcal{F}\left(\left\langle\mathcal{E}_{\gamma}\right\rangle, B\right)$ is a decreasing function over $B$ and $\left\langle\mathcal{E}_{\gamma}\right\rangle$. When the radiation reaction is negligible, $\mathcal{F}\left(\left\langle\mathcal{E}_{\gamma}\right\rangle, B\right)$ tends to 1 and the standard expression for the piston velocity, $\beta_{P}=B /(1+B)$, as used in Refs. [28] and [4], is obtained. The formula relating piston velocity to reflection coefficient 


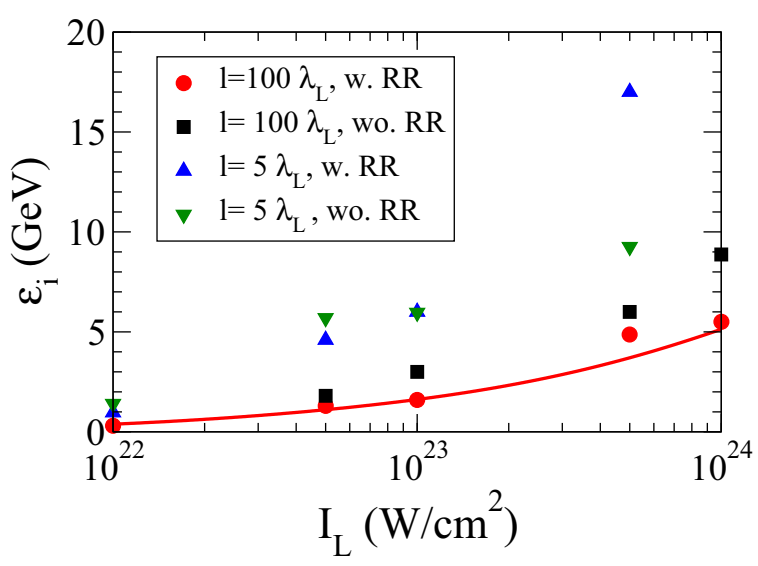

FIG. 7. (Color online) Laser intensity dependence of the maximum energy of piston-accelerated (reflected) deuterons. The red line corresponds to the analytical model calculations with $\mathrm{RR}$, respectively, for $l=100 \lambda_{L}$. The symbols are simulation results for given parameters.

[Eq. (19)] is valid in 1D only. Noncollinear motion breaks the correlation between energy conservation [Eq. (14)] and momentum conservation [Eq. (13)]. Accounting for the emission of high energy synchrotron radiation in the energy conservation results in a reduction to the piston velocity, which is brought out by the function $\mathcal{F}\left(\left\langle\mathcal{E}_{\gamma}\right\rangle, B\right)$. Dividing Eq. (13) by $\left(1-\beta_{p}\right)$ results in an equation governing the partition of the total absorbed laser energy between photons $\left(\eta_{\gamma}\right)$ and ions $\left(\eta_{i}\right)$ :

$$
\eta_{\text {total }}=\underbrace{\frac{\left\langle\mathcal{E}_{\gamma}\right\rangle}{2\left(1-\beta_{p}\right)}}_{\eta_{\gamma}}+\underbrace{\frac{2 B \mathcal{F}\left(\left\langle\mathcal{E}_{\gamma}\right\rangle, B\right)}{1+B\left(1+\mathcal{F}\left(\left\langle\mathcal{E}_{\gamma}\right\rangle, B\right)\right)}}_{\eta_{i}} .
$$

Figure 7 shows the energy of the reflected ions, $\varepsilon_{i}=$ $2 m_{i} c^{2} \gamma_{p}^{2} \beta_{p}^{2}$, as a function of laser intensity, as calculated using the expression for $\beta_{p}$ given in Eq. (20). The model results are in good agreement with the ion energy spectrum shown in Fig. 4(f), as obtained from the simulations for $I_{L}=1.1 \times 10^{23} \mathrm{~W} / \mathrm{cm}^{2}$. With increasing intensity a larger fraction of the laser energy is converted to high energy synchrotron radiation, leading to a larger reduction in the maximum ion energy. This tendency is confirmed in both the model calculations and simulation results.

\section{The light-sail regime}

Given that the RR force enhances ion acceleration in thin, relativistically transparent targets and has the opposite effect for thick targets for which hole-boring-RPA dominates, we now consider an intermediate case, $l=5 \lambda_{L}$, where $P_{s}$ has approximately the same magnitude as $P_{r}$. It is for this condition that the highest energy ions are achieved, as shown in Fig. 4(e). This arises from the fact that the laser ponderomotive force pushes almost all of the target electrons in the forward direction.
This case is close to the laser piston regime described in Ref. [2], where the ions can reach relativistic energies if the condition $E_{\|} \simeq 2 \pi e n_{e} l<E_{L}$ is fulfilled. Although the target considered here is thicker than that considered in the laser piston scenario in Ref. [2], the density is lower, resulting in a similar areal density. The reflection coefficient of the laser pulse in the present simulation is low compared to the idealized piston or the light-sail-RPA scenario, and the electrons are heated more, which decreases the energy transfer to ions. In addition, due to the electron forward motion, the RR force does not strongly affect the electron dynamics, and thus ion acceleration (at intensity equal to $1.1 \times 10^{23} \mathrm{~W} / \mathrm{cm}^{2} ; a_{L}=200$ ). At higher intensities, $\sim 5 \times 10^{23} \mathrm{~W} / \mathrm{cm}^{2}$, the condition $P_{r}>P_{s}$ is fulfilled, which is similar to the case of a thin foil (for example, the case of $l=0.8 \lambda_{L}$ at $1.1 \times 10^{23} \mathrm{~W} / \mathrm{cm}^{2}$ considered above). In this regime RR strongly enhances the laser piston efficiency and thus ion energy. This is observed when comparing the results with and without RR included in Fig. 7. These features are also in good agreement with Ref. [30], where the authors show with $3 \mathrm{D}$ numerical simulations that the RR force has limited effect on ion acceleration in this regime.

\section{DISCUSSION}

In summary, the effects of RR on the energy spectrum of accelerated ions in ultraintense laser-plasma interactions is shown to depend strongly on the target thickness (effectively areal density) and thus the underlying ion acceleration mechanism. Whereas the maximum ion energy is enhanced and a spectral peak produced in the case of relativistically transparent targets, in the hole-boring-RPA regime more than $30 \%$ of the laser energy is converted into intense synchrotron radiation, which reduces the piston velocity and thus the ion energy by a factor of 2 . An expression for the piston velocity that takes into account the radiation losses is derived for the first time. RR is found to have little effect on ion acceleration in the light-sail-RPA regime.

The sensitivity of the influence of RR reaction to the ion mass is determined by comparing the results for protons and deuterons in Figs. 4(d)-4(f). In all three regimes of target thickness explored, the ion mass influences the extent to which RR occurs, but does not change the overall physical processes discussed above. At $1.1 \times 10^{23} \mathrm{~W} / \mathrm{cm}^{2}$ the maximum ion energies obtained with a proton plasma are higher and lower than for a deuteron plasma for the $l=5 \lambda_{L}$ and $l=100 \lambda_{L}$ cases, respectively. The spectral distribution is also more peaked in the case of protons and $l=5 \lambda_{L}$, which is a signature of a transition to a light-sail-like RPA scenario. For a thick target, it has been shown that the electrostatic field increases with the ion mass and thus enhances synchrotron generation [20], which explains why the RR-force has a larger affect on deuterons than on protons. Nevertheless, it does significantly change the proton energy spectrum. Cryogenic target technology is being developed for the production of thin targets of pure hydrogen and deuterium. It should therefore be possible to experimentally investigate the effects explored in this paper at the multi-PW laser facilities presently under development. 


\section{ACKNOWLEDGMENTS}

We thank Prof. Y. Sentoku for use of the PICLS code. We acknowledge helpful advice from Prof. V. T. Tikhonchuk and Dr. E. d'Humières. This work is supported by EPSRC (Grants No. EP/J003832/1, No. EP/K022415/1 and No.
EP/L000237/1 - the UK Plasma HEC Consortium). Simulations were also performed using the ARCHIE-WeST high performance computer. Data associated with research published in this paper is accessible at http://dx.doi.org/ 10.15129/61fba9a0-7054-47e6-8199-9b20d0fcf997.
[1] A. Macchi, M. Borghesi, and M. Passoni, Rev. Mod. Phys. 85 751 (2013).

[2] T. Esirkepov, M. Borghesi, S. V. Bulanov, G. Mourou, and T. Tajima, Phys. Rev. Lett. 92, 175003 (2004).

[3] A. Macchi, S. Veghini, and F. Pegoraro, Phys. Rev. Lett. 103, 085003 (2009).

[4] A. P. L. Robinson, P. Gibbon, M. Zepf, S. Kar, R. G. Evans, and C. Bellei, Plasma Phys. Control. Fusion 51, 024004 (2009).

[5] M. Grech, S. Skupin, A. Diaw, T. Schlegel, and V. T. Tikhonchuk, New J. Phys. 13, 123003 (2011).

[6] A. Zhidkov, J. Koga, A. Sasaki, and M. Uesaka, Phys. Rev. Lett. 88, 185002 (2002).

[7] A. Di Piazza, K. Z. Hatsagortsyan, and C. H. Keitel, Phys. Rev. Lett. 102, 254802 (2009).

[8] M. Tamburini, F. Pegoraro, A. Di Piazza, C. H. Keitel, and A. Macchi, New J. Phys. 12, 123005 (2010).

[9] M. Chen, A. Pukhov, T. P. Yu, and Z. M. Sheng, Plasma Phys. Control. Fusion 53, 014004 (2011).

[10] R. Capdessus, E. d'Humières, and V. T. Tikhonchuk, Phys. Rev. E 86, 036401 (2012).

[11] N. M. Naumova, T. Schlegel, V. T. Tikhonchuk, C. Labaune, I. V. Sokolov, and G. Mourou, Phys. Rev. Lett. 102, 025002 (2009).

[12] I. V. Sokolov, J. Exp. Theor. Phys. 109, 207 (2009).

[13] I. V. Sokolov, N. M. Naumova, and J. A. Nees, Phys. Plasmas 16, 093115 (2009).

[14] I. V. Sokolov, J. A. Nees, V. P. Yanovsky, N. M. Naumova, and G. A. Mourou, Phys. Rev. E 81, 036412 (2010).

[15] L. D. Landau and E. M. Lifschitz, The Classical Theory of Fields, 4th ed. (Pergamon, New York, 1994), Vol. 2.
[16] S. C. Wilks, W. L. Kruer, M. Tabak, and A. B. Langdon, Phys. Rev. Lett. 69, 1383 (1992).

[17] R. Capdessus, M. Lobet, E. d'Humières, and V. T. Tikhonchuk, Phys. Plasmas 21, 123120 (2014).

[18] R. Capdessus, Ph.D. thesis, Dymamique d'un plasma non collisionnel interagissant avec une impulsion laser ultra-intense, University of Bordeaux, France, 2013.

[19] Y. Sentoku and A. Kemp, Comput. Phys. 227, 6846 (2008).

[20] R. Capdessus, E. d'Humières, and V. T. Tikhonchuk, Phys. Rev. Lett. 110, 215003 (2013).

[21] C. P. Ridgers, J. G. Kirk, R. Duclous, T. G. Blackburna, C. S. Brady, K. Bennett, T. D. Arber, and A. R. Bell, J. Comp. Phys. 260, 273 (2014).

[22] A. R. Bell and J. G. Kirk, Phys. Rev. Lett. 101, 200403 (2008); C. P. Ridgers et al., Phys. Plasmas 20, 056701 (2013).

[23] A. I. Nikishov and V. I. Ritus, Sov. Phys. JETP 19, 5 (1964).

[24] A. I. Nikishov and V. I. Ritus, Sov. Phys. JETP 29, 1 (1969).

[25] I. V. Sokolov, N. M. Naumova, J. A. Nees, and G. A. Mourou, Phys. Rev. Lett. 105, 195005 (2010).

[26] M. Tamburini, F. Pegoraro, A. DiPiazza, C. H. Keitel, T. V. Liseykina, and A. Macchi, Nucl. Instrum. Meth. B 653, 181 (2011).

[27] C. S. Brady, C. P. Ridgers, T. D. Arber, A. R. Bell, and J. G. Kirk, Phys. Rev. Lett. 109, 245006 (2012).

[28] T. Schlegel, N. Naumova, V. T. Tikhonchuk, C. Labaune, I. V. Sokolov, and G. Mourou, Phys. Plasmas 16, 083103 (2009).

[29] M. C. Levy, S. C. Wilks, M. Tabak, and M. G. Baring, Phys. Plasmas 20, 103101 (2013).

[30] M. Tamburini, T. V. Liseykina, F. Pegoraro, and A. Macchi, Phys. Rev. E 85, 016407 (2012). 